\title{
Low Estimated Glomerular Filtration Rate Is Prevalent among North Korean Refugees in South Korea
}

\author{
Young-Soo Song ${ }^{1}$, Seong-Woo Choi ${ }^{2, *}$ \\ 'Department of Health Science, Graduate School of Chosun University, Gwangju, Korea \\ ${ }^{2}$ Department of Preventive Medicine, Chosun University College of Medicine, Gwangju, Korea
}

Background: The number of North Korean refugees entering South Korea is rising. Few studies have investigated the risk of non-communicable disease in North Korean refugees. Moreover, kidney insufficiency, a risk factor for cardiovascular disease, has not been studied in this population. We compared the prevalence of noncommunicable disease and kidney function in North Korean refugees and South Koreans.

Methods: Our study was conducted using a case-control design. We enrolled 118 North Korean refugees from the Hana Center and selected 472 randomly sampled South Korean individuals as controls, who were age- and sexmatched with the North Korean refugees in a ratio of 1:4, from the 2014 Korea National Health and Nutrition Examination Survey database.

Results: The prevalence of non-communicable disease did not differ significantly between the groups; however, a low estimated glomerular filtration rate (eGFR; $<90 \mathrm{~mL} / \mathrm{min}$ per $1.73 \mathrm{~m}^{2}$ ) was more prevalent in the North Korean refugees than in the South Korean population $(52.1 \%$ vs. $29.9 \%, \mathrm{P}<0.001)$. After adjusting for covariates and weight gain after escape, the prevalence of a low eGFR was associated with the length of residence in South Korea (odds ratio, 2.84; 95\% confidence interval, 1.02-7.89).

Conclusion: The prevalence of non-communicable disease did not differ between North Korean refugees and the South Korean population, while a low eGFR was more prevalent in North Korean refugees than in South Koreans. Moreover, after adjusting for other covariates, the prevalence of a low eGFR in North Korean refugees was associated with the length of residence in South Korea.

Keywords: North Korean Refugees; Glomerular Filtration Rate; Chronic Renal Insufficiency

Received: January 20, 2017, Revised: March 6, 2017, Accepted: March 6, 2017

*Corresponding Author: Seong-Woo Choi https://orcid.org/0000-0002-6150-3934

Tel: +82-62-230-6344, Fax: +82-62-225-8293, E-mail: jcsw74@hanmail.net 


\section{INTRODUCTION}

Cardiovascular disease (CVD) is the main cause of mortality and morbidity in most parts of the world. ${ }^{1)}$ Consequently, methods for estimating the risk of CVD are needed to reduce both its incidence and disease burden. Decreased kidney function is an independent risk factor for $\mathrm{CVD}^{2)}$ and is correlated with cardiovascular mortality and morbidity. $^{3,4)}$

Immigrants to Western countries are often at high risk for CVD. ${ }^{5)}$ Investigations have found that individuals who migrate to countries that are more industrialized and wealthier than their birth country are more prone to non-communicable diseases (NCD) than the host population as a result of changes in lifestyle, such as diet and physical activity. ${ }^{6)}$ However, there are some limitations due to ethnic differences between immigrants and the host population.

Korea was an ethnically homogeneous nation for over 5,000 years and has been divided into North and South Korea for 70 years. South Korea is one of the most developed countries in the world, whereas North Korea has experienced economic hardship. ${ }^{7)}$ Since the 1990s, an increasing number of North Koreans (NK) have fled their country because of severe food deprivation, due in turn to economic failures and natural disasters. ${ }^{7)}$ Most NK refugees seek asylum in China or South Korea. The number of NK refugees in South Korea reached 29,543 in June 2016. ${ }^{8)}$ Most of the previous health-related studies focusing on these refugees have focused on their mental health, ${ }^{9)}$ growth status, ${ }^{10)}$ and infectious diseases, such as tuberculosis and hepatitis. ${ }^{11)}$ In comparison, few studies have investigated risk factors for CVD in NK refugees. To our knowledge, only one previous cohort study examined NCD in NK refugees. ${ }^{12)}$ Moreover, renal insufficiency, a known risk factor for CVD, has not been examined in this population.

Therefore, this study compared the prevalence of NCD and CVD risk factors, including renal insufficiency, between NK refugees and a South Koreans (SK), which are ethnically homogenous, and investigated the factors related to renal insufficiency in NK refugees.

\section{METHODS}

\section{Study Subjects}

A case-control study was performed. The cases were NK refugees and the controls were SK individuals. NK refugees were recruited from among those registered at the Hana Center in Gwangju between May and June 2014. There are 23 Hana Centers (government run resettlement centers) throughout South Korea that provide practical and specific aid required by NK refugees. Of the 569 NK refugees registered at the Hana Center in Gwangju, 138 agreed to participate in our study. Of those, 20 were excluded because no blood sample was available $(n=18)$ or they refused to complete the survey $(n=2)$; consequently, 118 NK refugees were enrolled in the study (19 males, 99 females). The SK subjects were sampled randomly and age- and sex-matched with the NK refugees in a ratio of 1:4 from the 2014 Korea National Health and Nutrition Examination Survey (KNHANES) database, a cross-sectional, nationally representative survey with a multistage, stratified sampling design conducted by the Korea Centers for Disease Control and Prevention. The study protocol was reviewed and approved by the Institutional Review Board of Chosun University Hospital (IRB approval no., Chosun 2014-04-014-005). Informed consent was obtained from all subjects. In total, 590 subjects (118 NK refugees, 472 SK people) were enrolled in our study.

\section{Measurements}

Trained examiners interviewed patients using a questionnaire that included items on smoking status, alcohol intake, physical activity, history of medications for diabetes, hypertension, and hyperlipidemia, weight gain since exiting North Korea, and length of residence in North Korea, in a third country, and in South Korea. Smoking status was based on self-reported cigarette use: never-smokers had smoked fewer than 100 cigarettes in their lifetime, and participants who had smoked 100 or more cigarettes were classified as past or current smokers based on their current smoking habits. Alcohol intake was assessed according to the participants' drinking behavior during the month before the interview. Physically active was indicated as 'yes' when the participant performed moderate or strenuous exercise on a regular basis (less than 30 minutes at a time more than 5 times a week for moderate exercise and more than 20 minutes at a time more than 3 times a week for strenuous exercise) or walked more than 30 minutes at a time than 5 times a week.

Weight was measured to the nearest $0.1 \mathrm{~kg}$ while the subjects were dressed in light clothing. Height was measured to the nearest $0.1 \mathrm{~cm}$ in stocking feet. Body mass index (BMI) was calculated as weight in $\mathrm{kg}$ divided by the square of height in meters. Waist circumference was measured using a flexible tape at the narrowest point between the lowest rib and the uppermost lateral border of the right iliac crest. General obesity was defined as BMI $\geq 25.0 \mathrm{~kg} / \mathrm{m}^{2}$ and abdominal obesity was defined as waist circumference $\geq 90 \mathrm{~cm}$ in men and $\geq 85 \mathrm{~cm}$ in women. Blood pressure was measured after the subject had rested for 5 minutes in a sitting position. Three readings each of systolic and diastolic blood pressure were recorded, and the average value of the second and third readings was used in the analyses. Hypertension was defined as systolic blood pressure $\geq 140 \mathrm{~mm} \mathrm{Hg}$ or diastolic blood pressure $\geq 90$ $\mathrm{mm} \mathrm{Hg}$, or taking antihypertensive medication. Blood samples were drawn from an antecubital vein following a 12-hour overnight fast, serum was separated within 30 minutes, and the samples were stored at $-70^{\circ} \mathrm{C}$ until analysis. Fasting blood glucose levels were measured using enzymatic methods and evaluated using an automated analyzer (Model 7600 Chemical Analyzer; Hitachi, Tokyo, Japan). Diabetes was defined as fasting serum glucose $\geq 126 \mathrm{mg} / \mathrm{dL}$ or taking insulin or oral diabetes medication, and hyperlipidemia was defined as total cholesterol $>240 \mathrm{mg} / \mathrm{dL}$, triglycerides $>200 \mathrm{mg} / \mathrm{dL}$, or high-density lipoprotein (HDL) cholesterol $<40 \mathrm{mg} / \mathrm{dL}$.

Kidney function was assessed according to the estimated glomerular filtration rate (eGFR), calculated using the Modification of Diet in Renal Disease formula ${ }^{13)}$ as follows: $186.3 \times\left(\right.$ serum creatinine $\left.e^{-1.154}\right) \times$ 
$\left(\right.$ age $\left.^{-0.203}\right)[\times 0.742$ if female $]$ with the serum creatinine concentration expressed in $\mathrm{mg} / \mathrm{dL}$. Low eGFR was defined as eGFR $<90 \mathrm{~mL} / \mathrm{min}$ per $1.73 \mathrm{~m}^{2}$, based on reports that even a mildly reduced eGFR was associated with CVD. ${ }^{14)}$ Urinary albumin and creatinine concentrations were measured using a turbidimetric immunoassay and the Jaffe method with a Hitachi-7600 analyzer (Hitachi). Albuminuria was defined according to the albumin/creatinine ratio (ACR), which was calculated by dividing the urinary albumin concentration $(\mu \mathrm{g})$ by the urinary creatinine concentration (mg).

\section{Statistical Analyses}

The results are expressed as mean \pm standard deviation or as percentages for categorical variables. After applying the Kolmogorov-Smirnov test to assess the normality of distributions, non-normally distributed data were log-transformed before the analysis. Student t-tests or chisquare were used to compare data of NK refugees and the SK participants, who were age- and sex-matched with the NK refugees in a ratio of 1:4 from the KNHANES database. Multiple logistic regression analysis was performed to assess the prevalence of low eGFR in NK refugees. Weight gain since migrating from North Korea and length of residence in North Korea, in a third country, and in South Korea were recorded dichotomously by the median value of each variable. Model 1 was adjusted for sex, age, waist circumference, smoking status, alcohol intake, physical activity, diabetes, hypertension, hyperlipidemia, albu- minuria, and length of residence in South Korea. Model 2 was also adjusted for weight gain since escape, and length of residence in North Korea and in a third country. The SPSS ver. 15.0 (SPSS Inc., Chicago, IL, USA) was used to conduct the statistical tests, and P-values $<0.05$ were considered statistically significant.

\section{RESULTS}

\section{General Characteristics of North Korean Refugees and South Korean Participants}

Table 1 shows the characteristics of the NK refugees and SK participants enrolled in the study. Height, weight, waist circumference, physical activity, systolic blood pressure, diastolic blood pressure, fasting glucose, hemoglobin Alc, triglycerides, HDL cholesterol levels, eGFR, and ACR were lower in NK refugees than in SKs. BMI was higher in NK refugees than in SKs. In NK refugees, weight gain since migrating from North Korea was $5.6 \pm 8.0 \mathrm{~kg}$ and the length of residence in North Korea, in a third country, and in South Korea was $34.4 \pm 11.8,5.3 \pm 4.6$, and $6.7 \pm 2.9$ years, respectively.

\section{Comparison of Disease Status between North Korean Refugees and South Korean Participants}

Table 2 shows the disease status of the NK refugees and SKs. The rate of general obesity was significantly higher in NK refugees than in SK

Table 1. Characteristics of North Korean refugees and South Koreans

\begin{tabular}{|c|c|c|c|}
\hline Characteristic & North Korean refugees & South Korean people & P-value \\
\hline Total no. & $118(20.0)$ & $472(80.0)$ & \\
\hline Male & $19(16.1)$ & $76(16.1)$ & \\
\hline Age (y) & $44.4 \pm 11.8$ & $44.3 \pm 11.8$ & $0.379^{\star}$ \\
\hline Height (cm) & $154.4 \pm 7.5$ & $160.1 \pm 7.5$ & $<0.001^{*}$ \\
\hline Weight $(\mathrm{kg})$ & $57.7 \pm 9.2$ & $59.9 \pm 10.8$ & $0.048^{*}$ \\
\hline Body mass index $\left(\mathrm{kg} / \mathrm{m}^{2}\right)$ & $24.2 \pm 3.2$ & $23.3 \pm 3.5$ & $0.010^{*}$ \\
\hline Waist circumference (cm) & $74.4 \pm 7.9$ & $78.4 \pm 9.7$ & $<0.001^{\star}$ \\
\hline Smoking & $14(11.9)$ & $61(13.0)$ & 0.103 \\
\hline Alcohol intake & $84(71.2)$ & $350(74.3)$ & 0.281 \\
\hline Physically active $^{\dagger}$ & $8(6.8)$ & $67(14.4)$ & 0.015 \\
\hline Systolic blood pressure (mm Hg) & $104.7 \pm 17.2$ & $111.9 \pm 13.8$ & $<0.001^{\star}$ \\
\hline Diastolic blood pressure (mm Hg) & $65.8 \pm 11.5$ & $73.9 \pm 9.6$ & $<0.001^{*}$ \\
\hline Hypertensive medication & $14(11.9)$ & $64(13.6)$ & 0.761 \\
\hline Fasting glucose (mg/dL) & $75.8 \pm 16.5$ & $97.8 \pm 23.2$ & $<0.001^{*}$ \\
\hline Hemoglobin A1C & $5.3 \pm 0.4$ & $5.6 \pm 0.7$ & $<0.001^{*}$ \\
\hline Diabetes medication & $5(4.2)$ & $30(6.4)$ & 0.514 \\
\hline Total cholesterol (mg/dL) & $182.7 \pm 38.0$ & $187.9 \pm 33.5$ & $0.083^{*}$ \\
\hline Triglycerides (mg/dL) & $85.1 \pm 106.7$ & $125.5 \pm 117.4$ & $<0.001^{\star}$ \\
\hline High-density lipoprotein cholesterol (mg/dL) & $52.1 \pm 10.6$ & $55.6 \pm 13.0$ & $0.012^{*}$ \\
\hline Estimated glomerular filtration rate $\left(\mathrm{mL} / \mathrm{min}\right.$ per $\left.1.73 \mathrm{~m}^{2}\right)$ & $88.6 \pm 15.4$ & $99.7 \pm 17.4$ & $<0.001^{*}$ \\
\hline Albumin creatinine ratio ( $\mu \mathrm{g} / \mathrm{mg}$ creatinine) & $12.4 \pm 28.3$ & $15.3 \pm 103.5$ & $0.020^{\star}$ \\
\hline Weight gain from escape $(\mathrm{kg})$ & $5.6 \pm 8.0$ & & \\
\hline Length of residence in North Korea (y) & $34.4 \pm 11.8$ & & \\
\hline Length of residence in a third country $(y)$ & $5.3 \pm 4.6$ & & \\
\hline Length of residence in South Korea (y) & $6.7 \pm 2.9$ & & \\
\hline
\end{tabular}

Values are presented as number (\%) or mean \pm standard deviation.

${ }^{*}$ P-value for log-transformed data. ${ }^{\dagger}$ Subjects who performed 30 minutes or more of moderate activity at least 5 days a week or 20 minutes of vigorous physical activity at least 3 days a week or walked more than 30 minutes at a time more than 5 times a week were regarded as doing physical activity. 
Table 2. Comparison of disease status between North Korean refugees and South Koreans

\begin{tabular}{|c|c|c|c|}
\hline Variable & $\begin{array}{l}\text { North Korean } \\
\text { refugees }\end{array}$ & $\begin{array}{l}\text { South Korean } \\
\text { people }\end{array}$ & P-value \\
\hline General obesity* & $43(36.8)$ & $126(26.7)$ & 0.031 \\
\hline Abdominal obesity ${ }^{\dagger}$ & $9(7.7)$ & 87 (18.4) & 0.005 \\
\hline Hypertension & $20(17.1)$ & $76(16.1)$ & 0.445 \\
\hline Diabetes & $5(4.2)$ & $34(7.2)$ & 0.171 \\
\hline Hyperlipidemia & $27(23.1)$ & $109(23.1)$ & 0.552 \\
\hline $\begin{array}{l}\text { Estimated glomerular filtration rate } \\
<90 \mathrm{~mL} / \mathrm{min} \text { per } 1.73 \mathrm{~m}^{2}\end{array}$ & $61(52.1)$ & $141(29.9)$ & $<0.001$ \\
\hline $\begin{array}{l}\text { Albumin creatinine ratio } \\
>30 \mu \mathrm{g} / \mathrm{mg} \text { creatinine }\end{array}$ & $9(7.7)$ & $31(6.6)$ & 0.397 \\
\hline
\end{tabular}

Values are presented as number (\%).

*Body mass index $\geq 25.0 \mathrm{~kg} / \mathrm{m}^{2}$. ${ }^{+}$Waist circumference $\geq 90 \mathrm{~cm}$ in men or $\geq 85 \mathrm{~cm}$ in women.

individuals ( $36.8 \%$ versus $26.7 \%, \mathrm{P}=0.031$ ). However, the prevalence of abdominal obesity was lower in NK refugees than in SKs (7.7\% versus $18.4 \%, \mathrm{P}=0.005)$. Low eGFR was more prevalent in NK refugees than in SKs (52.1\% versus $29.9 \%, \mathrm{P}<0.001)$. We found no significant betweengroup differences in hypertension, diabetes, hyperlipidemia, or albuminuria.

\section{Distribution of North Korean Refugees and South Korean Participants according to the Estimated Glomerular Filtration Rate}

Figure 1 shows the distribution of the NK refugees and SKs according to the eGFR based on a previous study. ${ }^{14)}$ A higher percentage of NK refugees had an eGFR $<90 \mathrm{~mL} / \mathrm{min}$ per $1.73 \mathrm{~m}^{2}$, whereas a higher percentage of SK people had an eGFR $\geq 90 \mathrm{~mL} / \mathrm{min}$ per $1.73 \mathrm{~m}^{2}$ (eGFR $<60$ : NK refugees $0.9 \%$, SKs $0.2 \%$; eGFR $60-74$ : NK refugees $18.8 \%$, SKs 4.7\%; eGFR 75-89: NK refugees 32.5\%, SKs 25.0\%; eGFR 90-104: NK refugees $32.5 \%$, SKs $36.4 \%$; and eGFR $\geq 105$ : NK refugees $15.4 \%$, SKs $33.7 \%)$.

\section{Comparison of Characteristics according to Estimated Glomerular Filtration Rate in North Korean Refugees}

Table 3 shows the characteristics of NK refugees according to the eGFR. Age, systolic blood pressure, diastolic blood pressure, fasting glucose, and length of residence in North Korea were significantly higher in those with eGFR $<90 \mathrm{~mL} / \mathrm{min}$ per $1.73 \mathrm{~m}^{2}$ than with eGFR $\geq 90 \mathrm{~mL} / \mathrm{min}$ per $1.73 \mathrm{~m}^{2}$.

\section{Odds Ratio for Low Estimated Glomerular Filtration Rate Prevalence among North Korean Refugees}

The odds ratios (ORs) for low eGFR prevalence among NK refugees are shown in Table 4. After adjusting for covariates (sex, age, waist circumference, smoking, drinking, physical activity, hypertension, diabetes, hyperlipidemia, albuminuria, and the length of residence in South Korea), the prevalence of low eGFR was significantly associated with the length of residence in South Korea (OR, 2.53; 95\% confidence interval [CI], 1.03-6.21). After also adjusting for weight gain since escape,

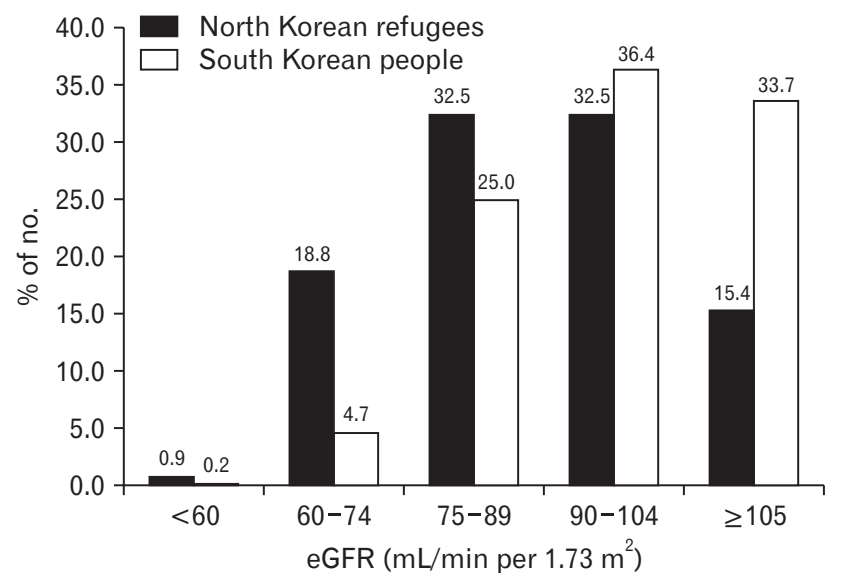

Figure 1. Distribution of the North Korean refugees and South Koreans according to the eGFR. eGFR, estimated glomerular filtration rate.

and length of residence in North Korea and in a third country, the association between low eGFR and length of residence in South Korea remained significant (OR, 2.84; 95\% CI, 1.02-7.89).

\section{DISCUSSION}

We compared the prevalence of NCD and low eGFR in NK refugees with that of the SK individuals. The prevalence of abdominal obesity was lower in NK refugees and the prevalence of NCD did not differ between groups; however, low eGFR was more prevalent in NK refugees than in SKs. Moreover, after adjusting for other covariates, the prevalence of low eGFR in NK refugees was associated with the length of residence in South Korea.

Previous studies have investigated the effects of immigration/seeking refuge on an individual's health by comparing the health status of immigrants/refugees with that of the host population. They have reported that individuals who migrate to more industrialized countries had a higher incidence of coronary heart disease than the host population as a result of acculturation. ${ }^{15,16)}$ However, there were some limitations to these studies because of the ethnic differences between the immigrants and host population. In the present study, it was possible to assess the effects of immigration/seeking refuge without any influence of genetic differences by comparing the health status of NK refugees and SK people, who are ethnically homogenous. SK people were randomly sampled from the KNHANES database, a nationally representative survey. Comparing SK people with individuals in the 2014 KNHANES database (2,516 males, 3,381 females), the SK people were younger ( $44.3 \pm 11.8$ versus $51.9 \pm 17.0$ years), included fewer males ( $16.1 \%$ versus $42.7 \%$ ), and had a lower eGFR $<90 \mathrm{~mL} / \mathrm{min}$ per $1.73 \mathrm{~m}^{2}$ (29.9\% versus $40.4 \%$ ) than the average subject in the 2014 KNHANES database.

The rate of obesity, as defined by the BMI, was significantly higher in the NK refugees than in SKs ( $36.8 \%$ versus $26.7 \%, \mathrm{P}=0.031$ ). However, the prevalence of abdominal obesity, as measured by waist circumfer- 
Table 3. Comparison of characteristics according to eGFR level in North Korean refugees

\begin{tabular}{|c|c|c|c|}
\hline \multirow{2}{*}{ Characteristic } & \multicolumn{3}{|c|}{ eGFR $\left(\mathrm{mL} / \mathrm{min}\right.$ per $\left.1.73 \mathrm{~m}^{2}\right)$} \\
\hline & $\geq 90$ & $<90$ & P-value \\
\hline Total no. & $57(48.3)$ & $61(51.7)$ & \\
\hline Male & $9(16.1)$ & $10(16.4)$ & 0.962 \\
\hline Age (y) & $40.6 \pm 9.1$ & $49.8 \pm 12.5$ & $<0.001^{*}$ \\
\hline Height (cm) & $153.5 \pm 7.1$ & $155.2 \pm 7.8$ & $0.235^{\star}$ \\
\hline Weight $(\mathrm{kg})$ & $56.2 \pm 7.5$ & $59.1 \pm 10.4$ & $0.137^{\star}$ \\
\hline Body mass index (kg/m²) & $23.9 \pm 3.0$ & $24.5 \pm 3.3$ & $0.340^{*}$ \\
\hline Waist circumference $(\mathrm{cm})$ & $73.4 \pm 7.2$ & $75.2 \pm 8.5$ & $0.267^{*}$ \\
\hline Smoking & $8(14.3)$ & $6(9.8)$ & 0.459 \\
\hline Alcohol intake & $44(78.6)$ & $40(65.6)$ & 0.119 \\
\hline Physically active $^{\dagger}$ & $4(7.1)$ & $4(6.6)$ & 0.900 \\
\hline Systolic blood pressure (mm Hg) & $101.2 \pm 15.0$ & $107.9 \pm 18.5$ & $0.046^{*}$ \\
\hline Diastolic blood pressure $(\mathrm{mm} \mathrm{Hg})$ & $63.6 \pm 11.6$ & $67.8 \pm 11.1$ & $0.034^{*}$ \\
\hline Hypertensive medication & $11(18.0)$ & $3(5.4)$ & 0.046 \\
\hline Fasting glucose (mg/dL) & $72.7 \pm 11.5$ & $78.7 \pm 19.8$ & $0.034^{*}$ \\
\hline Hemoglobin A1C & $5.3 \pm 0.3$ & $5.4 \pm 0.5$ & $0.183^{*}$ \\
\hline Diabetes medication & $3(4.9)$ & $2(3.6)$ & 0.719 \\
\hline Total cholesterol (mg/dL) & $177.1 \pm 36.2$ & $188.0 \pm 39.1$ & $0.116^{*}$ \\
\hline Triglycerides (mg/dL) & $85.8 \pm 137.7$ & $84.5 \pm 68.0$ & $0.569^{*}$ \\
\hline High-density lipoprotein cholesterol (mg/dL) & $50.6 \pm 9.7$ & $53.5 \pm 11.2$ & $0.156^{*}$ \\
\hline Albumin creatinine ratio ( $\mu \mathrm{g} / \mathrm{mg}$ creatinine) & $10.1 \pm 14.4$ & $14.5 \pm 36.7$ & $0.963^{*}$ \\
\hline Weight gain from escape $(\mathrm{kg})$ & $5.3 \pm 7.6$ & $5.9 \pm 8.4$ & $0.366^{*}$ \\
\hline Length of residence in North Korea (y) & $30.1 \pm 9.3$ & $38.6 \pm 12.4$ & $<0.001^{*}$ \\
\hline Length of residence in third country $(\mathrm{y})$ & $5.6 \pm 4.3$ & $5.0 \pm 4.9$ & $0.088^{*}$ \\
\hline Length of residence in South Korea (y) & $6.1 \pm 2.6$ & $7.2 \pm 3.1$ & $0.093^{*}$ \\
\hline
\end{tabular}

Values are presented as number (\%) or mean \pm standard deviation.

eGFR, estimated glomerular filtration rate.

${ }^{*}$ P-value for log-transformed data. ${ }^{\dagger}$ Subjects who performed 30 minutes or more of moderate activity at least 5 days a week or 20 minutes of vigorous physical activity at least 3 days a week or walked more than 30 minutes at a time more than 5 times a week were regarded as doing physical activity.

Table 4. Odds ratio for low estimated glomerular filtration rate prevalence among North Korean refugees

\begin{tabular}{|c|c|c|c|c|}
\hline \multirow{2}{*}{ Variable } & \multicolumn{2}{|c|}{ Model 1} & \multicolumn{2}{|c|}{ Model 2} \\
\hline & $\mathrm{OR}(95 \% \mathrm{Cl})$ & P-value & $\mathrm{OR}(95 \% \mathrm{Cl})$ & P-value \\
\hline Sex (female, male) & $0.57(0.06-5.78)$ & 0.633 & $0.84(0.07-10.08)$ & 0.892 \\
\hline Age (continuous) & $1.08(1.03-1.13)$ & 0.001 & $1.08(1.00-1.17)$ & 0.051 \\
\hline Waist circumference (continuous) & $1.00(0.94-1.06)$ & 0.975 & $1.02(0.95-1.10)$ & 0.593 \\
\hline Smoking (smoker, non-smoker) & $0.42(0.03-5.54)$ & 0.512 & $0.55(0.04-8.00)$ & 0.664 \\
\hline Alcohol drinking (drinking, non-drinking) & $0.69(0.23-2.04)$ & 0.503 & $0.64(0.20-2.12)$ & 0.469 \\
\hline Physically active (active, non-active) & $0.64(0.13-3.23)$ & 0.585 & $1.17(0.16-8.80)$ & 0.879 \\
\hline Hypertension (yes, no) & $2.90(0.71-11.88)$ & 0.139 & $4.86(0.83-28.32)$ & 0.079 \\
\hline Diabetes (yes, no) & $0.39(0.04-3.74)$ & 0.414 & $0.40(0.03-4.62)$ & 0.461 \\
\hline Hyperlipidemia (yes, no) & $0.61(0.19-1.90)$ & 0.389 & $0.46(0.12-1.70)$ & 0.243 \\
\hline Albuminuria (yes, no) & $0.89(0.13-5.99)$ & 0.903 & $2.11(0.17-26.44)$ & 0.564 \\
\hline Weight gain from escape $(\geq 4.5,<4.5 \mathrm{~kg})$ & & & $0.88(0.29-2.70)$ & 0.825 \\
\hline Length of residence in North Korea ( $\geq 33 \mathrm{y},<33 \mathrm{y})$ & & & $1.00(0.21-4.70)$ & 0.999 \\
\hline Length of residence in third country ( $\geq 5 \mathrm{y},<5 \mathrm{y}$ ) & & & $0.47(0.16-1.39)$ & 0.173 \\
\hline Length of residence in South Korea ( $\geq 5 \mathrm{y},<5 \mathrm{y}$ ) & $2.53(1.03-6.21)$ & 0.043 & $2.84(1.02-7.89)$ & 0.045 \\
\hline
\end{tabular}

Model 1: adjusted by sex, age, waist circumference, smoking, drinking, physical activity, hypertension, diabetes, hyperlipidemia, albuminuria, and length of residence in South Korea; model 2: adjusted by model 1 plus length of residence in North Korea, weight gain from escape, and length of residence in third country.

$\mathrm{OR}$, odds ratio; $\mathrm{Cl}$, confidence interval.

ence, was lower in the NK refugees than in the SKs $(7.7 \%$ versus $18.4 \%$, $\mathrm{P}=0.005)$. Because BMI can be readily calculated using height and weight data, it has been widely used as a measure of obesity; however, this measure has shortcomings. First, BMI is a poor measure in short subjects because short stature modifies the relationship between BMI and the prevalence of co-morbidities associated with obesity. ${ }^{17)}$ Moreover, universal BMI criteria are not suitable for the different Asian populations. ${ }^{18)}$ Indeed, it has been reported that waist circumference is a 
better predictor of coronary heart disease and type 2 diabetes than BMI. ${ }^{19)}$

Studies of immigrants in Western countries have found that the prevalence rates of obesity and NCD are lower in immigrants than in the host population, the so-called 'healthy immigrant effect.'. ${ }^{20)}$ In line with these studies, we found that measures of NCD, such as waist circumference, blood pressure, fasting glucose, hemoglobin Alc, and triglyceride levels, were lower in NK refugees than in the SK individuals. However, one should not conclude that the CVD risk is lower in NK refugees. Moreover, the development of a CVD prevention policy for NK refugees is important for several reasons. First, our study participants were refugees rather than ordinary immigrants, and as such, may have experienced a more burdensome migration because the process is often initiated by factors that drive the individual out of their country of origin. ${ }^{15)}$ A study found that refugees had higher all-cause and CVD mortality than family-reunified immigrants. ${ }^{21)}$ Second, although the prevalence of abdominal obesity was lower in NK refugees than in the SK participants, we found no between-group difference in the prevalence of NCD, and a higher prevalence of low eGFR, which indicates NK refugees might be more vulnerable to CVD. Third, NK refugees are in the lowest socioeconomic bracket in South Korea ${ }^{22)}$ and therefore have restricted access to healthcare services. ${ }^{23)}$

We found that low eGFR was more prevalent in NK refugees than in SK individuals. Assessment of immigrant health must consider the fact that their health is the result of risk factors accumulated over a lifetime, including the migration process before, during, and after immigration. ${ }^{24)} \mathrm{NK}$ refugees suffered from food shortages in North Korea, ${ }^{25)}$ and in other countries such as China. ${ }^{26)}$ Chronic malnutrition may reduce the glomerular filtration rate and alter the glomerular and tubular composition; these effects may persist after nutritional recovery, particularly in children. ${ }^{27)}$ Migration involves major changes that alter social, familial, and work arrangements and cause economic disruption, which may lead to mental stress. ${ }^{15,28)}$ Moreover, refugees are likely to have experienced serious stressors, such as war, torture, loss of family and friends, and long asylum procedures. ${ }^{15)}$ Such psychological stressors increase sympathetic nervous system activity and inflammatory cytokine levels, ${ }^{28)}$ which may impair kidney function.

Studies have shown that factors related to adaptation and integration into a new social and cultural environment may influence behavioral and CVD risk factors. ${ }^{16)}$ In a study of United States immigrant subgroups, length of residence in the United States was associated with a higher BMI. ${ }^{6)}$ In the Multi-Ethnic Study of Atherosclerosis, ${ }^{29)}$ the authors demonstrated that higher acculturation was associated with worse kidney function in Hispanics, and this might have been mediated by health behavior (but not by comorbidities).

However, our findings had some discrepancies. Despite the lack of a difference in NCD prevalence between the groups, and lower abdominal obesity in NK refugees, low eGFR was associated with the length of residence in South Korea. Although it is challenging to explain these discrepancies, there is a plausible explanation: the NK refugees might already have had a history of impaired kidney function while in North
Korea because of malnutrition and mental stress. A longer residence in South Korea, behavioral changes such as reduced physical activity, and a diet higher in sodium, might further reduce kidney function before the onset of obesity. The refugees in our study had resided in South Korea for an average of $6.7 \pm 2.9$ years and previous evidence suggests that, 15 years after migration, the prevalence of obesity in immigrants is similar to that in the host population. ${ }^{6)}$ In addition, NK refugees often rely on processed and fast-food meals, which frequently have a high salt content ${ }^{30}$ because they lack a primary meal preparer. ${ }^{22)}$ Consequently, they consume $3.8 \mathrm{~g}$ of sodium per day according to one study, which is higher than the World Health Organization recommendation $\left(<2.0\right.$ g per day) ${ }^{22)}$ Higher sodium intake is associated with a decline in eGFR in individuals with preserved kidney function. ${ }^{31)}$ Nevertheless, this finding might be a residual effect of insufficient adjustment for covariates or other variables that we did not measure.

The primary strengths of our study lie in the fact that NK refugees and SK people are ethnically homogenous, so it is possible to evaluate the effects of immigration/seeking refuge without considering genetic differences. However, several study limitations should also be considered. First, we used a cross-sectional design. Second, we did not obtain a direct measurement of glomerular filtration rate. Although inulin clearance is the gold standard, it was not feasible in a large epidemiological study. Third, we were unable to obtain detailed data on the medications used by the participants. Fourth, we were unable to obtain health-related data during their life in North Korea and after their escape from North Korea. Finally, because of the small number of NK refugees in our study, our results might reflect low statistical power. Further prospective studies are required to fully evaluate the association between low eGFR and related factors among NK refugees.

In conclusion, the prevalence of NCD did not differ between NK refugees and SK individuals. However, low eGFR was more prevalent in NK refugees than in SKs. Moreover, after adjusting for other covariates, the prevalence of low eGFR in NK refugees was associated with the length of residence in South Korea.

\section{CONFLICT OF INTEREST}

No potential conflict of interest relevant to this article was reported.

\section{REFERENCES}

1. Chimonas T, Fanouraki I, Liberopoulos EN, Chimonas E, Elisaf M. Diverging trends in cardiovascular morbidity and mortality in a low risk population. Eur J Epidemiol 2009;24:415-23.

2. National Kidney Foundation. K/DOQI clinical practice guidelines for chronic kidney disease: evaluation, classification, and stratification. Am J Kidney Dis 2002;39(2 Suppl 1):S1-266.

3. Muntner P, He J, Hamm L, Loria C, Whelton PK. Renal insufficiency and subsequent death resulting from cardiovascular disease in the United States. J Am Soc Nephrol 2002;13:745-53.

4. Shlipak MG, Simon JA, Grady D, Lin F, Wenger NK, Furberg CD, et al. Renal insufficiency and cardiovascular events in postmenopausal 
women with coronary heart disease. J Am Coll Cardiol 2001;38:705-11. 5. McDonald JT, Kennedy S. Is migration to Canada associated with unhealthy weight gain?: overweight and obesity among Canada's immigrants. Soc Sci Med 2005;61:2469-81.

6. Goel MS, McCarthy EP, Phillips RS, Wee CC. Obesity among US immigrant subgroups by duration of residence. JAMA 2004;292:2860-7.

7. Haggard S, Noland M. Famine in North Korea: markets, aid, and reform. New York (NY): Columbia University Press; 2009.

8. Ministry of Unification Republic of Korea. Current status of North Korean refugees' resettlement [Internet]. Seoul: Ministry of Unification Republic of Korea [cited 2016 Aug 18]. Available from: http://unikorea. go.kr/content.do?cmsid=3099.9.

9. Lee IS, Park HR, Kim YS, Park HJ. Physical and psychological health status of North Korean defector children. J Korean Acad Child Health Nurs 2011;17:256-63.

10. Schwekendiek D, Pak S. Recent growth of children in the two Koreas: a meta-analysis. Econ Hum Biol 2009; 7:109-12.

11. Dorman K, Bozinoff N, Redditt V, Kim E, Glazier RH, Rashid M. Health status of North Korean refugees in Toronto: a community based participatory research study. J Immigr Minor Health 2017;19:15-23.

12. Lee YH, Lee WJ, Kim YJ, Cho MJ, Kim JH, Lee YJ, et al. North Korean refugee health in South Korea (NORNS) study: study design and methods. BMC Public Health 2012;12:172.

13. Levey AS, Bosch JP, Lewis JB, Greene T, Rogers N, Roth D. A more accurate method to estimate glomerular filtration rate from serum creatinine: a new prediction equation. Modification of Diet in Renal Disease Study Group. Ann Intern Med 1999;130:461-70.

14. Levey AS, Coresh J. Chronic kidney disease. Lancet 2012;379:165-80.

15. Bo A, Zinckernagel L, Krasnik A, Petersen JH, Norredam M. Coronary heart disease incidence among non-Western immigrants compared to Danish-born people: effect of country of birth, migrant status, and income. Eur J Prev Cardiol 2015;22:1281-9.

16. Gadd M, Sundquist J, Johansson SE, Wandell P. Do immigrants have an increased prevalence of unhealthy behaviours and risk factors for coronary heart disease? Eur J Cardiovasc Prev Rehabil 2005;12:535-41.

17. Lara-Esqueda A, Aguilar-Salinas CA, Velazquez-Monroy O, GomezPerez FJ, Rosas-Peralta M, Mehta R, et al. The body mass index is a less-sensitive tool for detecting cases with obesity-associated co-morbidities in short stature subjects. Int J Obes Relat Metab Disord 2004; 28:1443-50.

18. Shiwaku K, Anuurad E, Enkhmaa B, Kitajima K, Yamane Y. Appropriate BMI for Asian populations. Lancet 2004;363:1077.
19. Lofgren I, Herron K, Zern T, West K, Patalay M, Shachter NS, et al. Waist circumference is a better predictor than body mass index of coronary heart disease risk in overweight premenopausal women. J Nutr 2004;134:1071-6.

20. McDonald JT, Kennedy S. Insights into the 'healthy immigrant effect': health status and health service use of immigrants to Canada. Soc Sci Med 2004;59:1613-27.

21. Norredam M, Olsbjerg M, Petersen JH, Juel K, Krasnik A. Inequalities in mortality among refugees and immigrants compared to native Danes: a historical prospective cohort study. BMC Public Health 2012; 12:757.

22. Choi SK, Park SM, Joung H. Still life with less: North Korean young adult defectors in South Korea show continued poor nutrition and physique. Nutr Res Pract 2010;4:136-41.

23. Wang BR, Kwon YD, Jeon W, Noh JW. Factors associated with the frequency of physician visits among North Korean defectors residing in South Korea: a cross-sectional study. BMC Health Serv Res 2015;15:90.

24. Spallek J, Zeeb H, Razum O. What do we have to know from migrants' past exposures to understand their health status?: a life course approach. Emerg Themes Epidemiol 2011;8:6.

25. Kim JE. Nutritional state of children in the Democratic People's Republic of Korea (DPRK): based on the DPRK final report of the National Nutrition Survey 2012. Pediatr Gastroenterol Hepatol Nutr 2014;17: 135-9.

26. Chang N, Kang EY, Lee JM, Lee MK. Anthropometric measurements and dietary patterns of North Korean migrant children in China. Korean J Nutr 2000;33:324-31.

27. Klahr S, Alleyne GA. Effects of chronic protein-calorie malnutrition on the kidney. Kidney Int 1973;3:129-41.

28. Bruce MA, Beech BM, Sims M, Brown TN, Wyatt SB, Taylor HA, et al. Social environmental stressors, psychological factors, and kidney disease. J Investig Med 2009;57:583-9.

29. Day EC, Li Y, Diez-Roux A, Kandula N, Moran A, Rosas S, et al. Associations of acculturation and kidney dysfunction among Hispanics and Chinese from the Multi-Ethnic Study of Atherosclerosis (MESA). Nephrol Dial Transplant 2011;26:1909-16.

30. Yon M, Lee Y, Kim D, Lee J, Koh E, Nam E, et al. Major sources of sodium intake of the Korean population at prepared dish level: based on the KNHANES 2008 \& 2009. Korean J Community Nutr 2011;16:47387.

31. Lin J, Hu FB, Curhan GC. Associations of diet with albuminuria and kidney function decline. Clin J Am Soc Nephrol 2010;5:836-43. 